\title{
An Energy-Aware Data Transmission Scheme under the Guarantee of Reliability for 3D WSNs
}

\author{
J. H. Zhang $\mathbb{D}^{1}{ }^{1}$ Z. X. Yi, ${ }^{1}$ and C. Y. Peng ${ }^{2}$ \\ ${ }^{1}$ School of Computer Science and Engineering, Central South University, Changsha 410083, China \\ ${ }^{2}$ School of Automation, Central South University, Changsha 410083, China \\ Correspondence should be addressed to J. H. Zhang; jinhuan_zhang@csu.edu.cn
}

Received 22 April 2020; Revised 19 July 2020; Accepted 4 September 2020; Published 28 September 2020

Academic Editor: Shahid Hussain

Copyright (c) 2020 J. H. Zhang et al. This is an open access article distributed under the Creative Commons Attribution License, which permits unrestricted use, distribution, and reproduction in any medium, provided the original work is properly cited.

\begin{abstract}
Three-dimensional wireless sensor networks (3D WSNs) play an important role to provide data collection services for Internet of things (IoT) in the real applications. However, many of the existing WSN data collection researches are based on a relatively simple linear or plane network model. The three-dimensional space problems are simplified to two-dimensional plane, which limits the applicability. In this paper, the data collection in 3D WSN is studied. In the three-dimensional space, we firstly analyze the data loads, energy consumption, and end-to-end (E2E) delay of each node when the network is following the shortest path routing. The mathematical analysis of data loads and E2E delay of each node are presented. Based on the analysis of data loads and energy consumption, an energy-ware data transmission scheme is proposed to achieve the trade-off optimization between the E2E delay and network lifetime under the guarantee of the transmission reliability. The key point of the proposed scheme is to make fully use of the unbalanced energy consumption of the 3D WSN. The performance of the proposed scheme is discussed, analyzed, and evaluated. The theoretical analysis and simulation results show that the E2E network delay and energy efficiency can be improved under the constraint of transmission reliability.
\end{abstract}

\section{Introduction}

Three-dimensional (3D) data collection is one of the key contents of Internet of things (IoT) [1-3]. Three-dimensional wireless sensor network (3D WSN), which is composed of many low-cost and low-power sensor nodes, is an important bridge of the integration of the physical world and the information world. Thus, 3D WSN is an important part of the data acquisition in IoT $[4,5]$. For example, many different types of sensors are widely deployed in different fields of environmental and ecological monitoring [6-9], health monitoring [10-12], home automation [13, 14], traffic control $[15,16]$, and coal mine production security [17], etc. They are applied to collect many data from the environment, traffic, terminals, and industry production for IoT.

However, data collection of 3D WSN faces the following challenges.
(1) Many of the existing WSN data collection researches are based on a relatively simple linear or plane network model. The research on 3D spatiotemporal data collection is mainly by extending the two-dimensional conclusion directly to the three-dimensional space. Or the three-dimensional space problems are simplified to two-dimensional plane. This limits the applicability [18-20]. Besides, the difficulty and computational complexity of data collection in 3D space of sensor network are much higher than that in 2D plane [21, $22]$. For example, the problem of network topology and geometry is extremely complex in 3D space. Thus, the computational complexity of $3 \mathrm{D}$ space algorithm is increased greatly. And, the problem of data collection with optimal solution in $2 \mathrm{D}$ plane may be a nondeterministic polynomial (NP) problem in $3 \mathrm{D}$ space 
(2) To reduce the end-to-end (E2E) delay and energy consumption under the guarantee of the transmission reliability is challenging in $3 \mathrm{D}$ wireless sensor networks. The data collection of 3D WSN is complicated because of the large-scale deployment, the limited energy of sensor nodes, and the unreliability of wireless communication. Some studies focus on improving the network transmission reliability, or reducing transmission delay, or improving the energy efficiency and prolonging the network lifetime. How to reduce the energy consumption of nodes and prolong the network lifetime while reducing the delay and ensuring the reliability of data transmission is an important problem to be effectively solved in 3D WSN data collection

To address the above issues, the mathematical expression of the data loads and E2E delay of each node in 3D WSN is presented. Then, an energy-aware data collection scheme for 3D WSN is proposed in the paper. The trade-off optimization of reducing energy consumption, reducing time delay, and improving reliability is obtained in the proposed scheme. The main contributions of the paper are as follows:

(1) The data loads and E2E delay of each node in 3D WSN are analyzed. In the paper, a 3D WSN is considered, which is a more realistic and suitable assumption in the real applications. In the three-dimensional space, we analyze the data loads, energy consumption, and E2E delay of each node. The mathematical model of data loads and E2E delay of each node is statistically presented

(2) An energy-aware data transmission scheme achieving the trade-off optimization between the E2E delay and the network lifetime with the guarantee of transmission reliability is proposed for $3 \mathrm{D} \mathrm{WSN}$. The key point of the proposed scheme is to make fully use of the unbalanced energy consumption of the 3D WSN. Specifically, for the nodes with more residual energy, they send multiple-packet copies consecutively to ensure transmission reliability, and they do not need to wait for the returned acknowledgement (ACK) to reduce the waiting time and transmission delay of each hop. For the nodes with more data loads and energy consumption, they adopt the overtime retransmission scheme to reduce the energy consumption. That is, the node sends the packet and then waits for the ACK from the receiver, and it will retransmit the packet when the transmitted packet is lost and the time is out

(3) The validity of the mathematical analysis is demonstrated through comparison between theoretical and simulation results. And the performance of the proposed scheme is discussed, analyzed, and evaluated. The theoretical analysis and simulation results show that tradeoff optimization of energy and E2E network delay under the constraint of transmission reliability can be achieved

The structure of this paper is organized as follows: the related works are reviewed in Section 2. Section 3 depicts the system model and assumptions adopted in the paper.
The mathematical model of data loads and E2E delay of each node in 3D WSN are presented in Section 4. Based on the analysis, an energy-aware data transmission scheme to achieve the trade-off optimization with the guarantee of transmission reliability is proposed for 3D WSN in Section 5 , including performance analysis. The simulation results are described and analyzed in Section 6 to evaluate the theoretical analysis and the proposed scheme. Finally, the paper concludes in Section 7.

\section{Related Works}

Several studies have already been conducted to analyze and characterize the energy consumption and energy hole phenomena for 2D WSN. The energy consumption and energy hole phenomena are characterized for 2D WSN by applying theoretical analysis in Refs. [23, 24]. The analytical results are applied to $2 \mathrm{D} \mathrm{WSN}$ routing and nodes deployment in order to balance the energy consumption and improve the network lifetime in Ref. [25]. However, 3D WSN is more realistic and suitable in the real applications, such as in coal mine and under water applications. As far as we know, there are no energy consumption analysis for 3D WSN.

For reducing the cost energy, there are many existing studies, such as compressed sensing (CS), data aggregation, and sleep/awake scheduling. Compressed sensing theory is widely used to reduce the communication cost of wireless sensor nodes and realize information reconstruction [26, 27]. The matrix completion technique is applied to realize low redundancy data collection $[28,29]$. In the aspect of cluster-based data aggregation, a variety of data aggregation optimization algorithms for cluster head selection have been proposed successively to prolong the network lifetime, such as Refs. [30, 31]. By considering energy consumption and constraints of communication, Ref. [31] presented an optimal topology of sensors algorithm by applying a new mathematical model for fuzzy clustering in 3D WSN. Data aggregation can effectively reduce the energy consumption of nodes, but most of the existing energy-efficient data aggregation research focuses on minimizing the energy consumption of data collection protocol. The network lifetime may not be improved by reducing the energy consumption of the whole protocol [25]. It is interesting and effective to improve the network performance by decreasing the energy consumption of hotspots and making full use of the extra energy consumption of nonhotspots. Sleep/wake-up scheduling is also a typical mechanism in wireless sensor networks to optimize the energy consumption. In Ref. [32], a selfadaptive sleep/wake-up scheduling approach is proposed based on the reinforcement learning technique. In the scheme, each node can decide its own operation mode of sleep, listening, or transmission in each time slot.

There are some studies which focus on reducing transmission delay or ensuring transmission reliability besides reducing the cost energy in data collection of 3D WSN. Ref. [33] proposed a fault-tolerant and energy-efficient broadcast routing protocol by partitioning the $3 \mathrm{D}$ network into a set of easily manageable clusters. In Ref. [34], the authors proposed 
a dynamic three-dimensional fuzzy routing based on traffic probability to enhance network lifetime and increase packet delivery ratio. To ensure the packet transmission reliability, timeout retransmission, packet reproduction, and broadcast schemes are widely adopted in packet transmission to the destination. However, packet reproduction and broadcast schemes may cause exhausted energy consumption. And timeout retransmission scheme requires extra time for ensuring packet transmission reliability. Therefore, to reduce the E2E delay and energy consumption under the guarantee of the transmission reliability is a challenging issue for data gathering in $3 \mathrm{D}$ wireless sensor networks.

In summary, there is no theoretical analysis to characterize the energy consumption for 3D WSN. Besides, how to achieve the trade-off optimization between the E2E delay and the network lifetime under the requirements of transmission reliability based on low hardware system configuration is one of the hot issues to be solved in the field.

\section{System Model and Problem Statements}

3.1. System Model. In the paper, we consider a WSN with $N$ sensor nodes uniformly and randomly scattered in the sphere. The radius and node density are assumed $R(\mathrm{~m})$ and $\rho\left(\mathrm{m}^{-3}\right)$, respectively. The sink node is placed at the centre of the sphere. Besides, the node transmission radius is assumed $r(\mathrm{~m})$. The transmission radius is unchanged. And nodes are no longer moving once deployed. The data is collected round by round. In one round of data collection, each node periodically generates one packet and transmitted it to the relaying node following the shortest path routing. Then, the packet is transmitted to the sink hop by hop. Due to the unreliability of wireless link in 3D WSN, the packet transmission is lossy, and we assume the successful probability of onehop transmission is $p$. All the nodes except the sink have the same initial energy. The energy of sink is assumed enough to gather all the data. Each node consumes energy for sending or receiving packets. For sending or receiving one-bit data, the cost energy is calculated as follows.

$$
\left\{\begin{array}{l}
E_{t}=E_{\mu}+\varepsilon_{\epsilon} d^{\alpha}, \\
E_{r}=E_{\mu},
\end{array}\right.
$$

where $d$ represents the distance between sending and relaying nodes. If it is less than the threshold, $\alpha=2$. Otherwise, $\alpha=4$. The other parameters' means and values can be found in Ref. [35].

3.2. Problem Statements. 3D WSN is more realistic and suitable in the real applications. However, many of the existing WSN data collection researches are based on a relatively simple linear or plane network model. As far as we know, there are no energy consumption analyses for 3D WSN. In addition, to reduce the E2E delay and energy consumption under the guarantee of the transmission reliability is a challenging issue for data gathering in 3D WSN. To address the issues, the meaning of the parameters is defined firstly.
In this paper, the end-to-end network transmission delay is named E2E delay. For a source node, it is defined as the time required from the packet initiated by the node to be successfully transmitted to the sink. For example, the source node $i$ with $h$ hops to the sink, if $\tau_{m}$ denotes the time delay of the $m$ th hop, the E2E delay of node $i$ is represented by $t_{i}$, and $t_{i}=\sum_{m=1}^{h} \tau_{m}$. Therefore, different source nodes may have different E2E delay. For the whole network, the E2E delay is defined as the maximum E2E delay of the source nodes in the 3D WSN. The objective of reducing E2E delay is to reduce the maximum E2E delay of the nodes in the $3 \mathrm{D}$ WSN. Thus, the mathematical expression is

$$
T=\min \left(\underset{0<i \leq n .}{\max }\left(t_{i}\right)\right)
$$

The network transmission reliability is defined as the proportion of the number of source nodes with generated packets successfully transmitted to the sink to the total number of nodes in the network. It is formulated as follows.

$$
\xi=\frac{\sum_{i=1}^{N} \mu_{i}}{N}
$$

where $\mu_{i}$ denotes whether the packet initiated by the source node $i$ successfully transmitted to the sink. When it succeeds, $\mu_{i}=1$. Otherwise, $\mu_{i}=0$. If $\zeta$ represents the network transmission reliability constraint to be satisfied, $\xi \geq \zeta$ is required.

For the nodes with less data loads, the energy consumption is relatively small. To fully utilize the residual energy of these nodes is meaningful while ensuring the network lifetime. In the paper, the network lifetime is defined as the time from the beginning of the network operation to the death of the first node. Thus, it depends on the node with the maximal energy cost in the network. To prolong the network lifetime means to reduce the maximal energy cost. Thus, we have the following equation.

$$
\eta=\min \left(\max \left(E_{\cos t}^{i}\right)\right),
$$

where $E_{\cos t}^{i}$ represents the energy consumption of node. Therefore, the trade-off problem could be expressed by the following equation.

$$
\left\{\begin{array}{l}
T=\min \left(\max _{0<i \leq n}\left(t_{i}\right)\right), \\
\eta=\min \left(\max \left(E_{\cos t}^{i}\right)\right),(i=1,2, \cdots, N), \\
\xi=\frac{\sum_{i=1}^{N} \mu_{i}}{N} \geq \zeta .
\end{array}\right.
$$

To summarize, the main purpose of the paper is to statistically analyze the data loads of each node in 3D WSN. Based on the analysis results, we want to achieve the trade-off optimization between the E2E network transmission delay and the energy efficiency under the constraint of the network reliability. 


\section{Analysis of Data Loads and E2E Delay for 3D WSN}

In the section, the data loads, energy consumption, and E2E delay of each node in the 3D WSN is statistically analyzed. In the analysis, the packets are forwarded following the shortest path routing. They are relayed hop by hop from the source nodes to the sink. Thus, the mathematical analysis is carried out in the hop-by-hop transmission mode.

4.1. Data Loads and E2E Delay of Each Node without Packet Loss. We firstly analyze the simplest case, that is, there is no packet loss in transmission. Following the shortest path routing, node $i$ with distance $l$ to the sink in 3D WSN will forward the packets from the source nodes with distance of $l+r, l+2 r$ $, \ldots, l+z r$ to the sink, where $l+z r \leq R$. Figure 1 shows the sketch of data forwarding.

Theorem 1. For the node $i$ with distance $l$ to the sink in a 3D $W S N$, the number of sending and receiving data packets is denoted, respectively, by $\epsilon_{r}(l)$ and $\epsilon_{t}(l)$. They are mathematically represented by the following equation.

$$
\left\{\begin{array}{l}
\epsilon_{r}(l)=\left\{\begin{array}{l}
\frac{\sum_{n=1}^{z}(l+n r)^{2}}{l^{2}}, \text { if } l+z r \leq R, \\
0, \text { if } l+r>R,
\end{array}\right. \\
\epsilon_{t}(l)=\epsilon_{r}(l)+1 .
\end{array}\right.
$$

Proof. The sphere is dived into many microelements, such as microhexahedrons. Considering the microhexahedron $C_{l}$ with the distance of $l(l=h r+x, x<r)$ to the sink, the microhexahedron is illustrated in Figure 2. As shown in Figure 2, the length of longitude direction is $l \sin \varphi$, the width of latitude direction is $l \sin \varphi d \theta$, and the radial height is $d l$, so the volume of the microhexahedron is

$$
d v=l^{2} \sin \varphi d l d \varphi d \theta
$$
$C_{l}$ is

Considering the node density $\rho$, the number of nodes in

$$
N_{C_{l}}=d v \bullet \rho=\rho l^{2} \sin \varphi d l d \varphi d \theta
$$

The nodes in $C_{l}$ need to receive and forward packets from the source nodes with distance of $l+r, l+2 r, \ldots, l+z r(l+z$ $r \leq R)$. Similarly, for the microhexahedron $C_{l+k r}$ with the distance of $l+k r(k=1,2, \cdots, z)$, the number of nodes is $N_{C_{l+k r}}$ $=\rho(l+k r)^{2} \sin \varphi d l d \varphi d \theta$. Therefore, the total number of receiving packets for the microhexahedron $C_{l}$ is

$$
\aleph_{l}=\rho\left[(l+r)^{2}+(l+2 r)^{2}+\cdots+(l+z r)^{2}\right] \sin \varphi d l d \varphi d \theta .
$$

We can get the following equation.

$$
\aleph_{l}=\rho \sum_{k=1}^{z}(l+k r)^{2} \sin \varphi d l d \varphi d \theta
$$

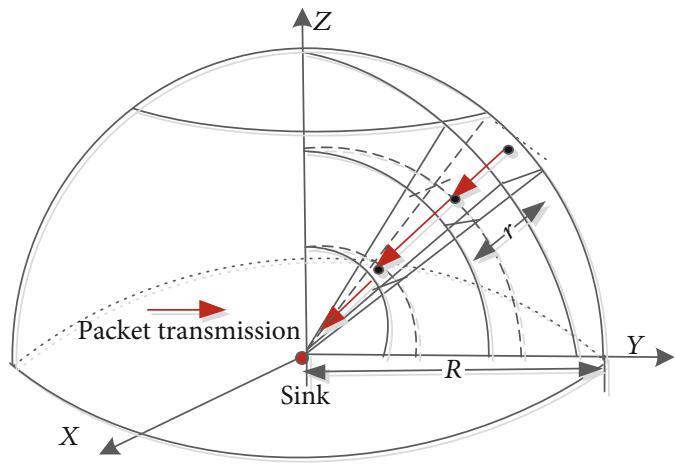

FIGURE 1: Sketch of data forwarding in 3D WSN.

Thus, for the nodes at the distance $l$, the average number of packets received by each node is

$$
\epsilon_{r}(l)=\frac{\aleph_{l}}{N_{C_{l}}}=\frac{\left\{\rho\left[(l+r)^{2}+(l+2 r)^{2}+\cdots+(l+z r)^{2}\right] \sin \varphi d l d \varphi d \theta\right\}}{\rho l^{2} \sin \varphi d l d \varphi d \theta} .
$$

After simplification, we have

$$
\epsilon_{r}(l)=\left\{\begin{array}{l}
\frac{\sum_{n=1}^{z}(l+n r)^{2}}{l^{2}}, \text { if } l+z r \leq R, \\
0, \text { if } l+r>R .
\end{array}\right.
$$

Because each node generates one data packet in one round of data gathering, the number of transmitted packets of a node at the distance $l$ is

$$
\epsilon_{t}(l)=\frac{\left[(l+r)^{2}+(l+2 r)^{2}+\cdots+(l+z r)^{2}\right]}{l^{2}}+1 .
$$

That is, $\epsilon_{t}(l)=\epsilon_{r}(l)+1$.

From the above equations, we can see that the data loads are smaller with the bigger $l$. Based on the above analysis, the energy cost of the node with distance $l$ to the sink is calculated by

$$
\begin{cases}E(l)=\epsilon_{r}(l) * E_{\text {elec }}+\epsilon_{t}(l) * E_{\text {elec }}+\epsilon_{t}(l) * \varepsilon_{f s} * x^{2}, & \text { if } x<d_{0} \text { and } z=0, \\ E(l)=\epsilon_{r}(l) * E_{\text {elec }}+\epsilon_{t}(l) * E_{\text {elec }}+\epsilon(l)_{t} * \varepsilon_{f s} * x^{4}, & \text { if } x \geq d_{0} \text { and } z=0, \\ E(l)=\epsilon_{r}(l) * E_{\text {elec }}+\epsilon_{t}(l) * E_{\text {elec }}+\epsilon_{t}(l) * \varepsilon_{f s} * r^{2}, & \text { if } r<d_{0} \text { and } z \neq 0, \\ E(l)=\epsilon_{r}(l) * E_{\text {elec }}+\epsilon_{t}(l) * E_{\text {elec }}+\epsilon_{t}(l) * \varepsilon_{f s} * r^{4}, & \text { if } r<d_{0} \text { and } z \neq 0,\end{cases}
$$

where $l=h r+x, x<r$.

If we assume the one-round transmission time for one hop is $\tau$, for the node with distance of $l$ to the sink, the E2E delay is $t_{h}=h \tau / 2$; therefore, the maximal network delay is $t=\max (h) \tau / 2$.

4.2. Data Loads and E2E Delay of Each Node in 3D WSN When Sending One Data Packet and One ACK Returned in Hop-by-Hop Mode. Because of the unreliable wireless links, 


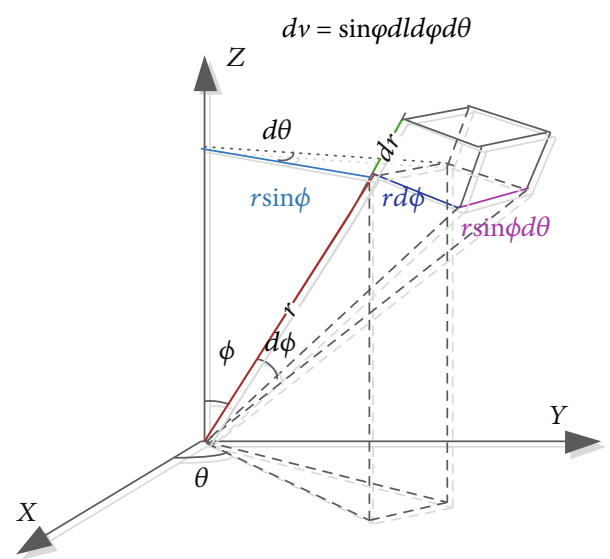

FIGURE 2: Illustration of the microhexahedron.

there are some packets lost in the transmission. To ensure the transmission reliability, the sender needs to wait for the returned ACK from the receiver to ensure the packets successfully transmitted. When the time is out, the sender does not receive the returned ACK; it will retransmit the packet.where $\sigma_{h+k, t}^{h+k}(\zeta)=\sigma_{h+k, t}^{h}(\zeta)=1-\left(1-p_{h+k} q_{h+k}\right)^{\gamma_{h+k}(\zeta)} / p_{h+k} q_{h+k}$ and $\gamma_{h+k}(\zeta)=\left\lceil\log \left(1-\zeta^{1 /(h+k)}\right) / \log \left(1-p_{h+k} q_{h+k}\right)\right\rceil . \quad \varphi_{t}(l)$ and $\varphi_{r}(l)$, respectively, represent the sending and receiving number of ACK packets.

Theorem 2. Under the required network transmission reliability $\zeta$, one-hop transmission reliability $p_{h}$, and the returned ACK transmission reliability $q_{h}$, the number of sending and receiving data packets and ACK packets is, respectively

$$
\left\{\begin{array}{l}
\epsilon_{t}(l)=\sum_{k=0}^{z} \sigma_{h+k, t}^{h}(\zeta) \frac{(l+k r)^{2}}{l^{2}}, \\
\epsilon_{r}(l)=\epsilon_{t}(l+r) p_{h+1}, \\
\varphi_{t}(l)=\epsilon_{r}(l), \\
\varphi_{r}(l)=\epsilon_{t}(l) p_{h} q_{h-1},
\end{array}\right.
$$

Proof. The maximum transmission number of packets for nodes at $l=h r+x$ is denoted by $\gamma_{h}(\zeta)$. And the required one-hop transmission reliability is $\zeta^{1 / h}$. We have $\zeta^{1 / h}=1$ $-\left(1-p_{h} q_{h}\right)^{\gamma_{h}(\zeta)}$. Hence, the following is obtained.

$$
\gamma_{h}(\zeta)=\left\lceil\frac{\log \left(1-\zeta^{1 / h}\right)}{\log \left(1-p_{h} q_{h}\right)}\right\rceil .
$$

According to Ref. [36], we get the number of transmitted packets statistically as the following equation.

$$
\sigma_{h, t}^{h}(\zeta)=\frac{1-\left(1-p_{h} q_{h}\right)^{\gamma_{h}(\zeta)}}{p_{h} q_{h}}
$$

Similarly, for the nodes with $h+j$ hops to the sink, the maximum transmission number and the sending number of packets are statistically obtained as follows.

$$
\begin{aligned}
& \gamma_{h+j}(\zeta)=\left[\frac{\log \left(1-\zeta^{1 /(h+j)}\right)}{\log \left(1-p_{h+j} q_{h+j}\right)}\right], \\
& \sigma_{h+j, t}^{h+j}(\zeta)=\sigma_{h+j, t}^{h}(\zeta)=\frac{1-\left(1-p_{h+j} q_{h+j}\right)^{\gamma_{h+j}(\zeta)}}{p_{h+j} q_{h+j}} .
\end{aligned}
$$

According to Section 4.1, for the node with distance of $l$ to the sink, it needs to send its own generated packet and forward the packets from the source nodes at the position with distance of $l+r, \ldots, l+z r$ to the sink. Therefore, the statistical sending packet number is $\epsilon_{t}(l)=\sigma_{h, t}^{h}(\zeta$ )$+\sigma_{h+1, t}^{h}(\zeta)\left((l+r)^{2} / l^{2}\right)+\sigma_{h+2, t}^{h}(\zeta)\left((l+2 r)^{2} / l^{2}\right)+\cdots+\sigma_{h+z, t}^{h}(\zeta$ )$\left((l+z r)^{2} / l^{2}\right)$. After simplification, we get

$$
\begin{aligned}
& \epsilon_{t}(l)=\sum_{k=0}^{z} \sigma_{h+k, t}^{h}(\zeta) \frac{(l+k r)^{2}}{l^{2}}, \\
& \epsilon_{r}(l)=\epsilon_{t}(l+r) p_{h+1} .
\end{aligned}
$$

That is $\epsilon_{r}(l)$ $=-$ $\sigma_{h+1, t}^{h+1}(\zeta) p_{h+1}\left((l+r)^{2} / l^{2}\right)+\sigma_{h+2, t}^{h+1}(\zeta) p_{h+1}\left((l+2 r)^{2} / l^{2}\right)+\cdots+$ $\sigma_{h+z, t}^{h+1}(\zeta) p_{h+1}\left((l+z r)^{2} / l^{2}\right)$. The sending number of ACKs equals to the number of receiving data packets, which is $\varphi_{t}(l)=\epsilon_{r}(l)$. And the number of receiving ACKs equals the number of receiving data packets of its next hop multiplied by $q_{h-1}$ considering the ACK loss in the transmission. It is $\varphi_{r}(l)=\epsilon_{t}(l) p_{h} q_{h-1}$.

If we assume the one-round transmission time for one hop is $\tau$, the time interval between each sending packet copies is $\chi$, and the one round trip time out is $l$, the E2E delay for the node with distance of $l$ to the sink is calculated by the following.

$$
t_{i}=h\left[\tau+\left(\sigma_{h, t}^{h}(\delta)-1\right) \iota\right]
$$

4.3. Data Loads and E2E Delay of Each Node When Sending One Data Packet and Returning n-ACKs in Hop-by-Hop Mode. In order to reduce the unnecessary energy cost caused by the ACK lost in packet retransmission scheme. Ref. [36] proposed the sending one packet and returning multi-ACKs transmission scheme. In the section, the data loads in 3D WSN adopting the sending one packet and returning multi-ACKs transmission scheme are statistically analyzed.where $\sigma_{h+k, t}^{h}(\zeta)$ $=1-\left(1-p_{h}\left(1-\left(1-q_{h}\right)^{n}\right)\right)^{\gamma_{h+k}(\zeta)} / p_{h}\left(1-\left(1-q_{h}\right)^{n}\right)$ and $\gamma_{h+k}$ $(\zeta)=\left\lceil\log \left(1-\zeta^{1 /(h+k)}\right) / \log \left(1-p_{h+k}\left(1-\left(1-q_{h+k}\right)^{n}\right)\right)\right\rceil$.

Theorem 3. For the nodes with distance $l=h r+x$ to the sink, the one-hop transmission reliability $\zeta^{1 / h}$ needs to be satisfied. Under the condition that the one-hop transmission 
reliabilities are, respectively $p_{h}$ and $q_{h}$ for data packet and $A C K$ packet, and there are $n$ returned ACKs, the number of sending and receiving data and ACK packets is, respectively

$$
\left\{\begin{array}{l}
\epsilon_{t}(l)=\sum_{k=0}^{z} \sigma_{h+k, t}^{h}(\zeta) \frac{(l+k r)^{2}}{l^{2}}, \\
\epsilon_{r}(l)=\sum_{k=1}^{z} \sigma_{h+k, t}^{h}(\zeta) p_{h+1} \frac{(l+k r)^{2}}{l^{2}} \\
\varphi_{t}(l)=n \bullet \epsilon_{r}(l), \\
\varphi_{r}(l)=n \bullet \epsilon_{t}(l) p_{h} q_{h-1},
\end{array}\right.
$$

Proof. Considering the maximum number of transmitting packets $\gamma_{h}(\zeta)$, the following equation is obtained.

$$
\zeta^{1 / h}=1-\left(1-p_{h}\left(1-\left(1-q_{h}\right)^{n}\right)\right)^{\gamma_{h}(\zeta)} .
$$

And we get

$$
\gamma_{h}(\zeta)=\left\lceil\frac{\log \left(1-\zeta^{1 / h}\right)}{\log \left(1-p_{h}\left(1-\left(1-q_{h}\right)^{n}\right)\right)}\right\rceil .
$$

Therefore, the statistical number of the packets sent by the source node to ensure the one-hop transmission reliability is calculated by

$$
\sigma_{h, t}^{h}(\zeta)=\frac{1-\left(1-p_{h}\left(1-\left(1-q_{h}\right)^{n}\right)\right)^{\gamma_{h}(\zeta)}}{p_{h}\left(1-\left(1-q_{h}\right)^{n}\right)} .
$$

Similarly, for the source nodes with $h+j$ hops to the sink, the transmission number of their own generated packet is

$$
\sigma_{h+j, t}^{h}(\zeta)=\sigma_{h+j, t}^{h+j}(\zeta)=\frac{1-\left(1-p_{h+j}\left(1-\left(1-q_{h+j}\right)^{n}\right)\right)^{\gamma_{h+j}(\zeta)}}{p_{h+j}\left(1-\left(1-q_{h+j}\right)^{n}\right)} .
$$

Besides, the data loads transmitted by nodes with distance $l$ to the sink also include the generated packets from the source nodes located in the area with the distances of $l$ $+r, l+2 r, \ldots, l+z r$ to the sink. Therefore, the number of transmitted and received packets is mathematically described as follows.

$$
\begin{aligned}
& \epsilon_{t}(l)=\sigma_{h, t}^{h}(\zeta)+\sigma_{h+1, t}^{h}(\zeta) \frac{(l+r)^{2}}{l^{2}}+\sigma_{h+2, t}^{h}(\zeta) \frac{(l+2 r)^{2}}{l^{2}}+\cdots+\sigma_{h+z, t}^{h}(\zeta) \frac{(l+z r)^{2}}{l^{2}} \\
& \epsilon_{r}(l)=\sigma_{h+1, t}^{h+1}(\zeta) p_{h+1} \frac{(l+r)^{2}}{l^{2}}+\sigma_{h+2, t}^{h+1}(\zeta) p_{h+1} \frac{(l+2 r)^{2}}{l^{2}}+\cdots+\sigma_{h+z, t}^{h+1}(\zeta) p_{h+1} \frac{(l+z r)^{2}}{l^{2}} .
\end{aligned}
$$

And because $n$-ACKs are returned for one successful received data packet, the number of sent and received ACKs is obtained.

$$
\begin{gathered}
\varphi_{t}(l)=n \bullet \epsilon_{r}(l), \\
\varphi_{r}(l)=n \bullet \epsilon_{t}(l) p_{h} q_{h-1} .
\end{gathered}
$$

If we assume the one-round transmission time for one hop is $\tau$, the time interval between each sent packet copy is $\chi$, and the time out for retransmitting is $\iota$, the E2E delay for the node with distance of $l$ to the sink is

$$
\begin{array}{r}
h\left[\left(\sigma_{h, t}^{h}(\zeta)-1\right) \iota+\frac{\tau}{2}+\left(\sigma_{h, t}^{h}(\zeta) p_{h+1} n-1\right) \chi+\frac{\tau}{2}\right] \\
\quad=h\left[\tau+\left[\left(\sigma_{h, t}^{h}(\zeta)-1\right) \iota+\left(\sigma_{h, t}^{h}(\zeta) p_{h} n-1\right) \chi\right]\right] .
\end{array}
$$

4.4. Data Loads and E2E Delay of Each Node by Sending Multiple-Packet Copies Consecutively without ACK in Hopby-Hop Mode

Theorem 4. For ensuring the transmission reliability, the number of transmitted packets for nodes with distance $l$ to the sink is calculated by the following equation.

$$
\left\{\begin{array}{l}
\epsilon_{t}(l)=\sum_{k=0}^{z} \lambda_{h+k}(\zeta) \frac{(l+k r)^{2}}{l^{2}}, \\
\epsilon_{r}(l)=\epsilon_{t}(l+r) p_{h+1},
\end{array}\right.
$$

where $\lambda_{h+k}(\zeta)=\left\lceil\log \left(1-\zeta^{1 /(h+k)}\right) / \log \left(1-p_{h+k}\right)\right\rceil$.

Proof. To ensure the transmission reliability, the number of sending packet copies for nodes with distance $l$ meets the following equation.

$$
\zeta^{1 / h}=1-\left(1-p_{h}\right)^{\lambda(\zeta)}
$$

Therefore, the number of packet copies sent consecutively without the returned ACK by source nodes with the distance $l$ to the sink are mathematically formulated as:

$$
\lambda_{h}(\zeta)=\left\lceil\frac{\log \left(1-\zeta^{1 / h}\right)}{\log \left(1-p_{h}\right)}\right\rceil .
$$

It means that each forwarding node transmits $\lambda_{h}(\zeta)$ packet copies to ensure the one-hop transmission reliability in the routing path to the sink. Thus, the number of packets forwarded and received by nodes with distance $l$ to the sink is obtained, respectively, as follows.

$$
\begin{aligned}
\epsilon_{t}(l)= & \lambda_{h}(\zeta)+\lambda_{h+1}(\zeta) \frac{(l+r)^{2}}{l^{2}}+\lambda_{h+2}(\zeta) \frac{(l+2 r)^{2}}{l^{2}} \\
& +\cdots+\lambda_{h+z}(\zeta) \frac{(l+z r)^{2}}{l^{2}} \\
\epsilon_{r}(l)= & \epsilon_{t}(l+r) p_{h+1}
\end{aligned}
$$

If there are multiple-packet copies transmitted to the next hop, the transmission is successful when the next hop 
successfully receives the first arrived packet. For the number of $\lambda_{h}(\zeta)$ packet copies, the probability of the first, second, and third packet copy successfully transmitted is $P(\mu=1)=p_{h}$, $P(\mu=2)=\left(1-p_{h}\right) p_{h}$, and $P(\mu=3)=\left(1-p_{h}\right)^{2} p_{h}$, respectively. In the same way, the probability of the $\lambda_{h}(\zeta)$ th packet copy successfully transmitted is $P\left(\mu=\lambda_{h}(\zeta)\right)=$ $\left(1-p_{h}\right)^{\lambda_{h}(\zeta)-1} p_{h}$. As described in the above section, $\tau$ denotes the hop transmission round time, and $\chi$ represents the interval time of transmission between packets or packet copies. For the source node with $h$ hops to the sink, the E2E delay is represented as the following equation.

$$
t_{h}=h\left[\sum_{i=1}^{\lambda_{h}(\zeta)}\left(1-p_{h}\right)^{i-1} p_{h}\left(\frac{\tau}{2}+(i-1) \chi\right)\right] .
$$

\section{An Energy-Aware Data Transmission Scheme under the Guarantee of Delay and Reliability}

From the statistical analysis, the overall trend of data loads is increasing with the nodes located in the region closer to the sink. Thus, nodes far from the sink have more residual energy. To fully use the residual energy to reduce the E2E delay and ensure the transmission reliability, an energyaware data transmission scheme is proposed. The proposed energy-aware data transmission scheme is aimed at improving the energy utilization of the nodes far from the sink and achieves the trade-off optimization between the E2E delay and the energy under the constraint of the network reliability.

5.1. The Overall Scheme. The overall of the proposed scheme is illustrated in Figure 3. As shown in the figure, the network is divided into near and far areas. For the source or relay nodes in the far area, there are multiple-packet copies which are transmitted consecutively without waiting for ACK. For nodes in the near area, they follow the overtime retransmission mechanism to conserve energy. The one-hop transmission time is reduced compared with the timeout retransmission scheme, because there is no need to wait for ACK or even retransmit the packet when timeout. Besides, the energy utilization of the nodes far from the sink is improved by sending multiple-packet copies consecutively.

5.2. The Design of the Proposed Scheme. The parameter $\delta$ is used to represent the partition of the network into the far and near regions. If the source nodes with distance $l$ to the sink are bigger than $\delta R$, the source nodes are in the far area and send multiple-packet copies consecutively without waiting for the return of ACK. The number of packet copies $\lambda_{h}(\zeta)$ is calculated following equation (31).

The multiple-packet copies are transmitted consecutively with the interval time $\chi$. When one of the packet copies is successfully transmitted to the next hop node, the one-hop transmission succeeds. Then, the packet is forwarded with the same number of copies until the next hop node with the distance is smaller than or equal to $\delta$

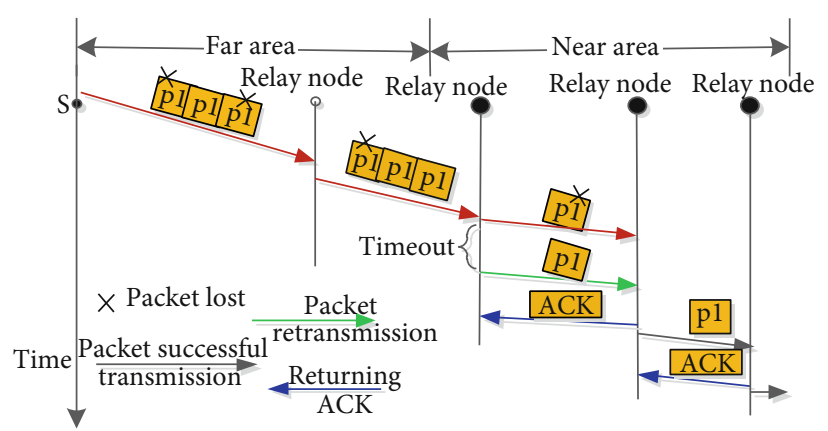

FIgURE 3: Sketch of the proposed scheme.

$R$. If the relay nodes with the distance is smaller than $\delta R$ , the packets are transmitted following the overtime retransmission scheme. The maximum number of transmissions is also $\lambda_{h}(\zeta)$ for forwarding nodes in near area. If the distance $l$ of the source nodes is smaller than $\delta R$, the source nodes adopt the overtime retransmission scheme. The maximum number of transmissions $\gamma_{h}(\zeta)$ is calculated as equation (23). After the source node sends one data packet to its next hop, it waits for the returned ACK packet. If it receives the ACK, the data packet is successfully transmitted to its next hop. If it does not receive the returned ACK caused by data packet loss or ACK loss, the source node will retransmit the lost packet after the one round trip timeout $t_{o}$. The retransmission is implemented until the data packet successfully transmitted to the next hop and the ACK received under the constraint of the maximum number of transmissions $\gamma_{h}(\zeta)$.

The detailed implementation is described in Algorithm 1.

5.3. Performance Analysis and Discussion. In the section, the performance of the proposed scheme for 3D WSN is statistically analyzed.

5.3.1. Data Loads. If the source node with distance $l$ is bigger than $\delta R$, the node sends packet copies consecutively with the number of $\lambda_{h}(\zeta)$ without waiting for the returned ACK. The source nodes in the location with distance of $l+r, l+2 r$ $, \ldots, l+z r$ adopt the same transmission scheme. They relay their generated data packet copies to the node with distance $l$ hop by hop. Therefore, the data load of the node with distance $l$ is obtained.

$$
\begin{gathered}
\epsilon_{t}(l)=\lambda_{h}(\zeta)+\lambda_{h+1}(\zeta) \frac{(l+r)^{2}}{l^{2}}+\lambda_{h+2}(\zeta) \frac{(l+2 r)^{2}}{l^{2}}+\cdots+\lambda_{h+z}(\zeta) \frac{(l+z r)^{2}}{l^{2}}, \\
\epsilon_{r}(l)=\epsilon_{t}(l+r) p_{h+1}, \\
\varphi_{t}(l)=\varphi_{r}(l)=0 .
\end{gathered}
$$

If the nodes with distance $l$ is less than $\delta R$, and we assume $l+9 r \geq \delta R$, we know that the nodes located in the region with the distance between $l$ and $l+(\vartheta-1) r$ adopt the overtime retransmission scheme which is sending one data packet and returning $n$-ACKs. If the node is a source node, it transmits its generated packet. In addition, it needs to forward 


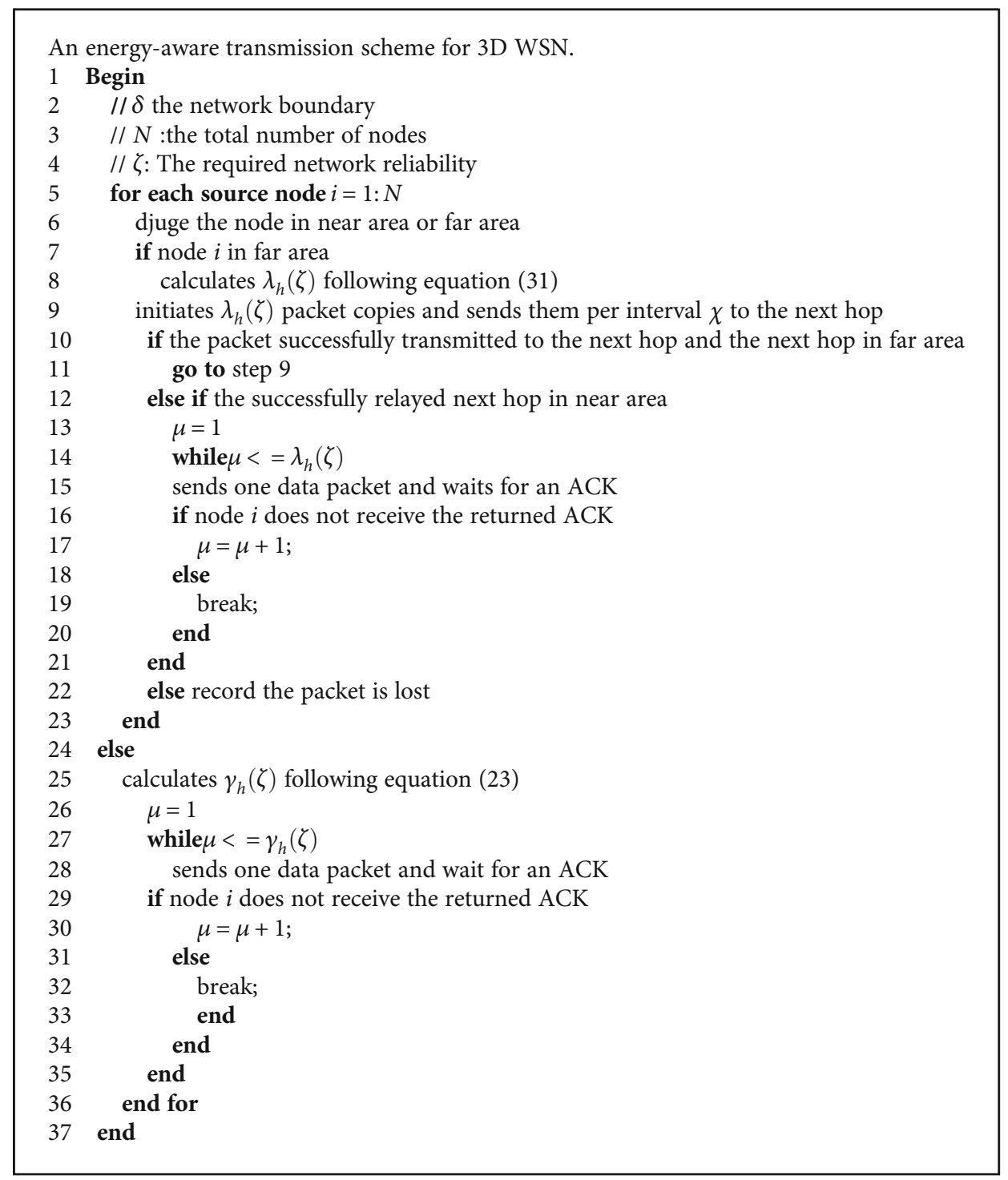

Algorithm 1.

data packets from the nodes in the regions with the distance of $l+r, l+2 r, \ldots, l+(\vartheta-1) r, l+\vartheta r, \ldots, l+z r$. Therefore, the statistical number of data packets needs to be transmitted and received is, respectively, as follows.

$$
\begin{aligned}
\epsilon_{t}(l)= & \sigma_{h, t}^{h}(\zeta)+\sigma_{h+1, t}^{h}(\zeta) \frac{(l+r)^{2}}{l^{2}}+\sigma_{h+\vartheta-1, t}^{h}(\zeta) \frac{(l+(\vartheta-1) r)^{2}}{l^{2}} \\
& +\lambda_{h+\vartheta}(\zeta) \frac{(l+\vartheta r)^{2}}{l^{2}}+\cdots+\lambda_{h+z}(\zeta) \frac{(l+z r)^{2}}{l^{2}} \\
\epsilon_{r}(l)= & \sigma_{h+1, t}^{h+1}(\zeta) p_{h+1} \frac{(l+r)^{2}}{l^{2}}+\sigma_{h+2, t}^{h+1}(\zeta) p_{h+1} \frac{(l+2 r)^{2}}{l^{2}} \\
& +\cdots+\sigma_{h+9-1, t}^{h+1}(\zeta) p_{h+1} \frac{(l+(\vartheta-1) r)^{2}}{l^{2}}+\epsilon_{t}(l+r) p_{h+1},
\end{aligned}
$$

where $\gamma_{h}(\zeta)=\left\lceil\log \left(1-\zeta^{1 / h}\right) / \log \left(1-p_{h}\left(1-\left(1-q_{h}\right)^{n}\right)\right)\right\rceil$ and $\sigma_{h, t}^{h}(\zeta)=\left(1-\left(1-p_{h}\left(1-\left(1-q_{h}\right)^{n}\right)\right)^{\gamma_{h}(\zeta)}\right) / p_{h}\left(1-\left(1-q_{h}\right)^{n}\right)$.

The number of sent and received ACK packets is

$$
\begin{gathered}
\varphi_{t}(l)=n \bullet \epsilon_{r}(l), \\
\varphi_{r}(l)=n \bullet \epsilon_{t}(l) p_{h} q_{h-1} .
\end{gathered}
$$

For a node with $h$ hops to the sink, we get the following comparison from the above results,

$$
\lambda_{h}(\zeta)=\left\lceil\frac{\log \left(1-\zeta^{1 / h}\right)}{\log \left(1-p_{h}\right)}\right\rceil>\gamma_{h}(\zeta)=\left\lceil\frac{\log \left(1-\zeta^{1 / h}\right)}{\log \left(1-p_{h}\left(1-\left(1-q_{h}\right)^{n}\right)\right)}\right\rceil(n \geq 1)
$$

$\gamma_{h}(\zeta)$ is the maximum number of one data packet transmissions to ensure the one-hop transmission reliability. This 
means that the node following the multiple-packet copy transmission has more data loads than that adopting the timeout retransmission. Therefore, for the nodes located in the far area, the multiple-packet copy transmission could improve the residual energy utilization under the guarantee of the transmission reliability and without influence on the network lifetime. And the timeout retransmission scheme adopted for the nodes located in near area could reduce energy cost compared with the application of multiplepacket copy transmission.

5.3.2. End-to-End Delay. If the source node with distance $l$ is bigger than $\delta R$, the $\mathrm{E} 2 \mathrm{E}$ delay is

$$
\begin{aligned}
t_{h}=\omega & {\left[\sum_{i=1}^{\lambda_{h}(\zeta)}\left(1-p_{h}\right)^{i-1} p_{h}\left(\frac{\tau}{2}+(i-1) \chi\right)\right]+(h-\omega) } \\
\cdot & {\left[\tau+\left[\left(\sigma_{h, t}^{h}(\zeta)-1\right) \iota+\left(\sigma_{h, t}^{h}(\zeta) p_{h} \alpha-1\right) \chi\right]\right], }
\end{aligned}
$$

where $\omega$ denotes the hops of the data packets needed to be relayed to the next relay node in the region with distance less than $\delta R$. If the node with distance $l$ is less than $\delta R$, the E2E delay is

$$
t_{h}=h\left[\tau+\left[\left(\sigma_{h, t}^{h}(\zeta)-1\right) \iota+\left(\sigma_{h, t}^{h}(\zeta) p_{h} \alpha-1\right) \chi\right]\right] .
$$

For the E2E delay of a node, we need to compare the onehop transmission time required between multiple-packet copy transmission scheme and the timeout retransmission scheme. We have $\tau+\left[\left(\sigma_{h, t}^{h}(\zeta)-1\right) \iota+\left(\sigma_{h, t}^{h}(\zeta) p_{h} \alpha-1\right) \chi\right]-$ $\sum_{i=1}^{\lambda_{h}(\zeta)}\left(1-p_{h}\right)^{i-1} p_{h}((\tau / 2)+(i-1) \chi)>(\tau / 2)+\left(\sigma_{h, t}^{h}(\zeta)-1\right) \iota$ $+\left(\sigma_{h, t}^{h}(\zeta) p_{h} \alpha-1\right) \chi+\left(\lambda_{h}(\zeta)-1\right) \chi>0$.

\section{Simulation Results and Analysis}

In the section, the mathematical analysis of data loads and E2E delay in 3D WSN are evaluated by comparison with the simulation results, and performance of the proposed scheme is validated. The evaluation on the performance of the proposed scheme includes the influence of parameters and comparison with the existed schemes. The simulation results are analyzed in detail.

6.1. Parameter Settings. In the simulation, a 3D spherical WSN with $N=5000$ nodes and $R=50(\mathrm{~m})$ is considered. In the network, sink is located at the center. Each node except sink with the same initial energy $1(\mathrm{~J})$ and the transmission radius is assumed $r=10(\mathrm{~m})$. Other parameters are shown in Table 1. The simulation is implemented hundreds of times. The average simulation result of one round of data collection is adopted to evaluate the performance.

6.2. Evaluation on the Mathematical Analysis. In the section, the mathematical analysis of data loads in 3D WSN is evaluated firstly. The validity is proved by comparing with the simulation results. The correlation coefficient between the theoretical and experimental results is applied to the quantitative evaluation on the fitting degree.

6.2.1. Evaluating the Mathematical Analysis of Data Loads and E2E Delay When Sending One Data Packet without Packet Loss. The mathematical analysis of data loads in 3D WSN is evaluated when following the sending one data packet without packet loss. Figures 4(a) and 4(b) show the comparison between the simulation results and the theoretical analysis. As shown in Figure 4(a), the overall trend of the number of the sending packet is increasing with the nodes closer to the sink, which is reflected by both the simulation and theoretical results. In addition, we can see that the number of sending packet of each node in theory is almost median line of the simulation. The difference between them is caused by the assumption in theory analysis that the nodes in 3D WSN are dense enough. The correlation coefficient between the theoretical and experimental results is 0.6306 . Therefore, the theoretical results are in reasonable agreement with the simulation results. The comparison of E2E delay in mathematical analysis and simulation is shown in Figure 4(b). The average error between the theoretical and simulation results is nearly 0 . The correlation coefficient between the theoretical and simulation results is nearly 1 . Therefore, the mathematical analysis of E2E delay in the 3D WSN is verified when sending one data packet without packet loss. Figure 5 describes the simulation results of energy cost of each node in the 3D WSN. The result shows that the energy cost increase as the nodes are closer to the sink, and the changing trends with distance to the sink is almost the same as that of the data loads.

6.2.2. Evaluating the Mathematical Analysis of Data Loads and E2E Delay When Sending One Data Packet with One ACK Returned in Hop-by-Hop Mode. The comparison of the simulation results and the theoretical results of data loads and E2E delay in the 3D WSN when sending one data packet with one ACK returned are shown in Figures 6(a) and 6(b). As shown in Figure 6(a), the overall increasing trend of the number of the sending packet is reflected by both the theoretical results and the simulation results. From the figure, we can see that the theoretical result of each node is the median line of the simulation results. The correlation coefficient between the theoretical and experimental results is 0.6190. The mathematical results agree with the simulation results to a certain extent. In Figure 6(b), the comparison of E2E delay between mathematical analysis and the simulation results is shown. The correlation coefficient between the theoretical and experimental results is 0.9898 . It shows that the simulation results are consistent with the theoretical results.

6.2.3. Evaluating the Mathematical Analysis of Data Loads and E2E Delay in 3D WSN When Sending One Data Packet and Returning $n$-ACKs. To evaluate the validity of the mathematical analysis of data loads and E2E delay in 3D WSN when sending one data packet and returning $n$-ACKs, we assume $n=2$ randomly. When sending one data packet and returning 2-ACKs, the validity of the theoretical analysis is 
TABle 1: Parameters' means and values.

\begin{tabular}{lcc}
\hline Parameter & Means & Value \\
\hline$d_{0}(\mathrm{~m})$ & Distance threshold & 87 \\
$E_{\text {elec }}(\mathrm{n} / / \mathrm{bit})$ & Transmitting circuit loss & 50 \\
$\varepsilon_{\mathrm{fs}}\left(\mathrm{p} J / \mathrm{bit} / \mathrm{m}^{2}\right)$ & Power amplification energy & 10 \\
$\varepsilon_{\mathrm{amp}}\left(\mathrm{p} / \mathrm{bit} / \mathrm{m}^{4}\right)$ & Power amplification energy & 0.0013 \\
$l_{\mathrm{p}}(\mathrm{bit})$ & Data packet length & 100 \\
$l_{\mathrm{a}}(\mathrm{bit})$ & ACK packet length & 10 \\
$\tau(\mathrm{ms})$ & Round-trip time & 50 \\
$\iota(\mathrm{ms})$ & Round-trip time to out \\
$\chi(\mathrm{ms})$ & The interval time for next packet transmission & 60 \\
\hline
\end{tabular}

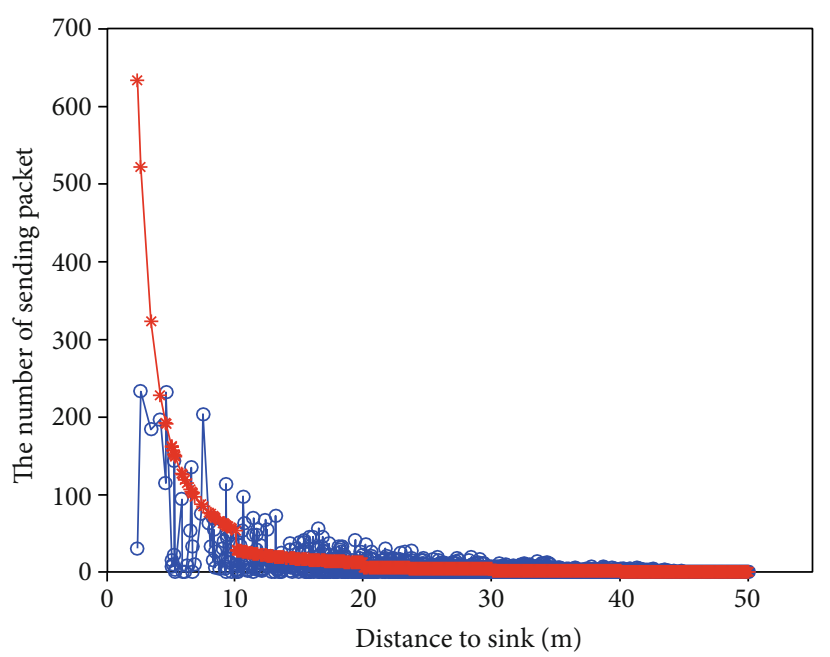

(a)

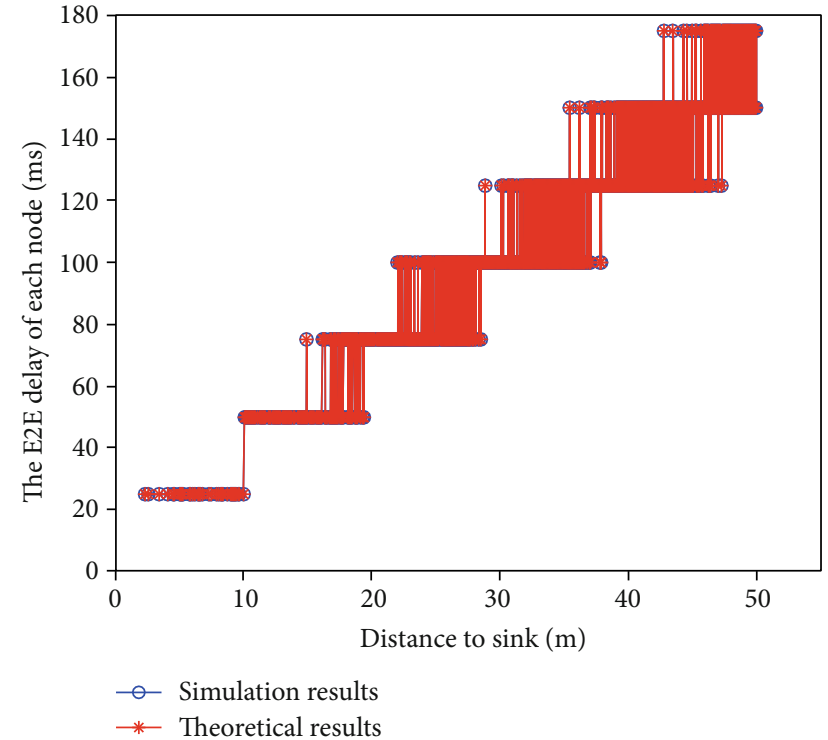

(b)

FIGURE 4: Comparison of the simulation results and the theoretical analysis in a 3D WSN. (a) The number of sending packet of each node. (b) The E2E delay of each node.

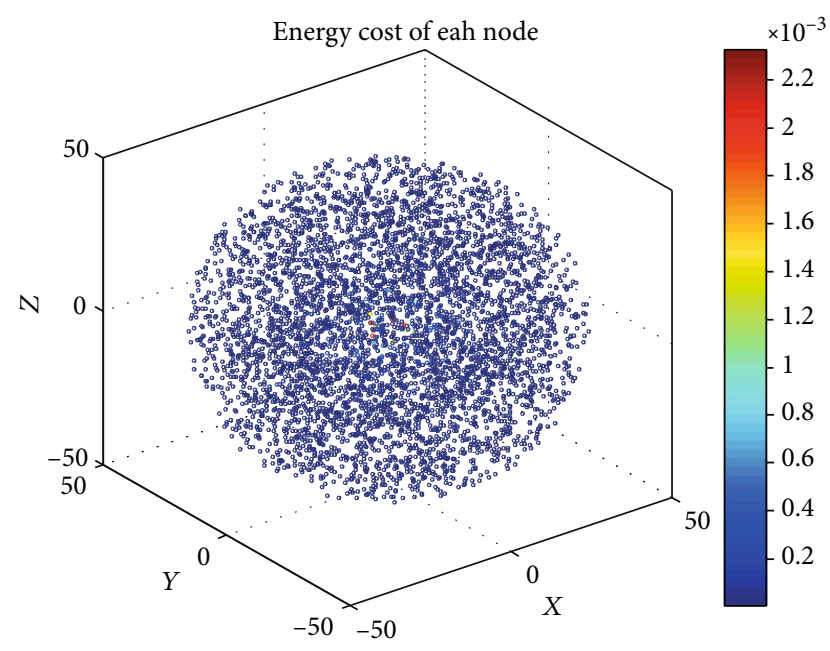

FIGURE 5: Energy cost in the 3D WSN. proved by comparing with the simulation results. Figures 7(a) and 7(b) show the comparison of the simulation and theoretical results in the 3D WSN. In Figure 7(a), both the theoretical results and the simulation results demonstrate the overall increasing trend of the number of the sending packet with the nodes closer to the sink. The correlation coefficient between the theoretical and experimental results is 0.6232. The difference between them is because that the nodes in 3D WSN are assumed dense enough in the mathematical characterizing. The comparison of E2E delay between the mathematical analysis and the simulation is shown in Figure 7(b). As can be seen from the figure, the mathematical analysis of E2E delay in $3 \mathrm{D}$ WSN is in consistent with the simulation results. The correlation coefficient between the theoretical and experimental results is 0.9929 .

6.2.4. Evaluating the Mathematical Analysis of Data Loads and E2E Delay in 3D WSN When Sending Multiple-Packet Copies without Returning ACK. When sending multiple- 


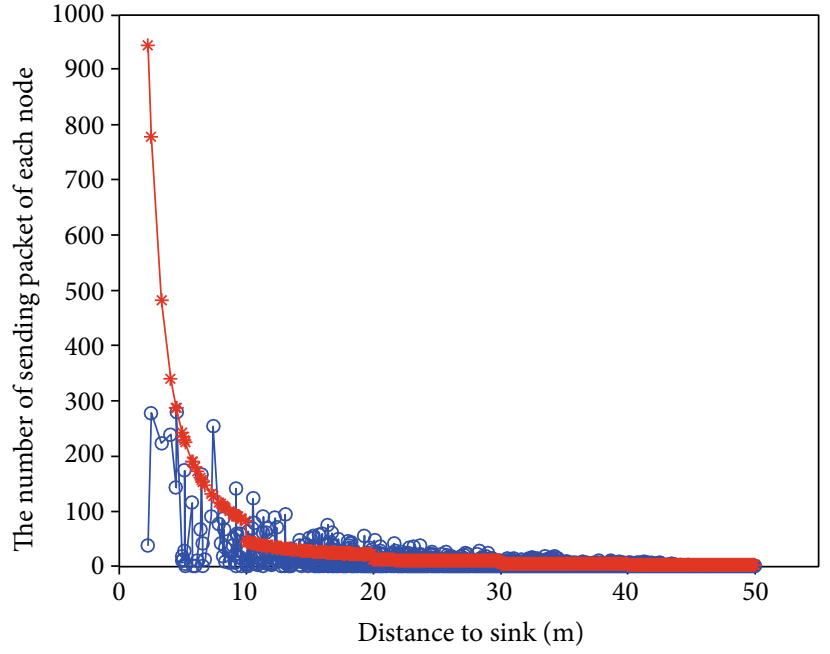

(a)

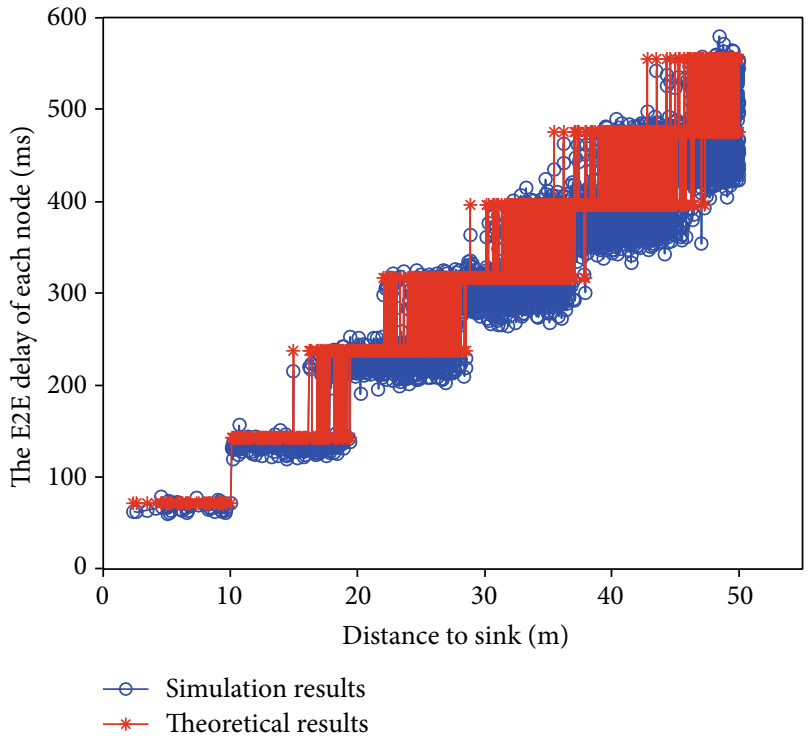

(b)

FIGURE 6: Comparison of the simulation results and the theoretical analysis in a 3D WSN. (a) The number of sending packet of each node. (b) The E2E delay of each node.

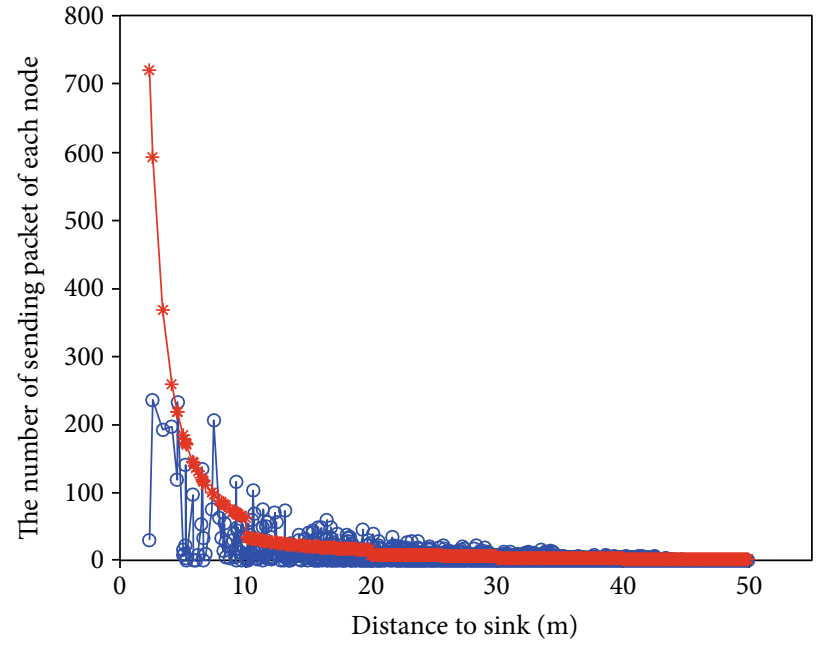

(a)

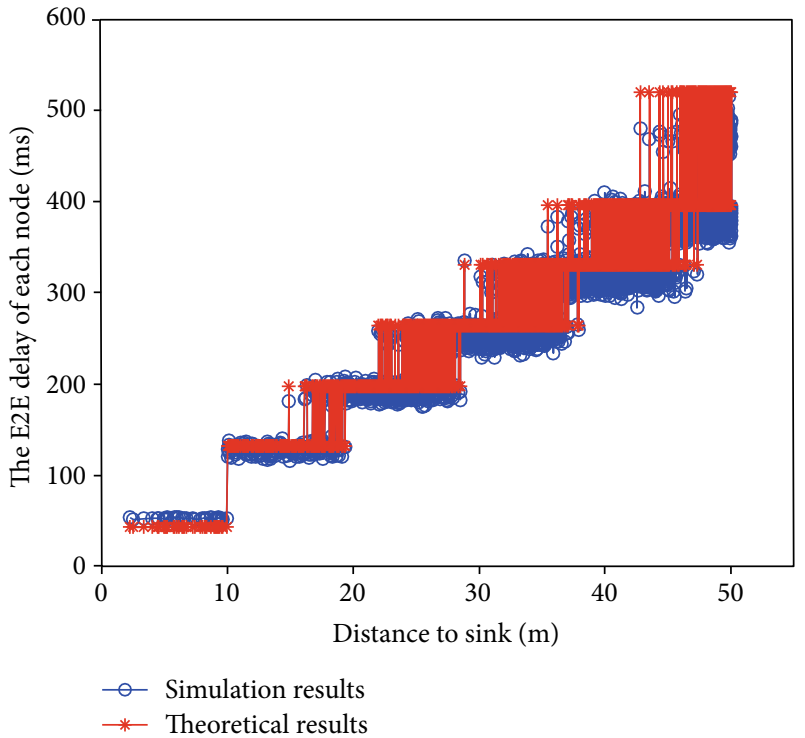

(b)

FIGURE 7: Comparison of the simulation results and the theoretical analysis in a 3D WSN. (a) The number of sending packet of each node. (b) The E2E delay of each node.

packet copies without returning ACK, the comparison of the simulation and the theoretical results in the 3D WSN is shown in Figures 8(a) and 8(b). As can be seen from Figure $8(\mathrm{a})$, the changing trend of the number of the sending packet is increasing with the nodes closer to the sink, which is reflected by both the theoretical analysis and the simulation results. From the figure, the theoretical result of each node is the median line of the simulation result. The correlation coefficient between the theoretical and experimental results is 0.6217 . The mathematical results are consistent with the simulation results. The comparison of E2E delay between mathematical analysis and simulation is demonstrated in Figure 8(b). The correlation coefficient between the theoreti$\mathrm{cal}$ and experimental results is 0.9992 . They are found to be in reasonable line with the simulation results.

6.3. Performance Evaluation on Different Parameters. In the part, the parameters influence on the proposed scheme are 


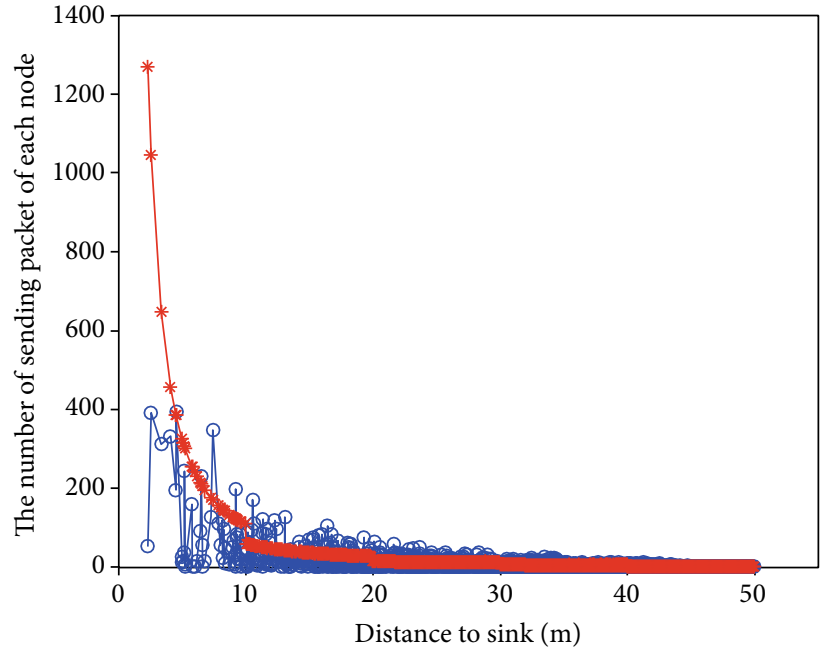

(a)

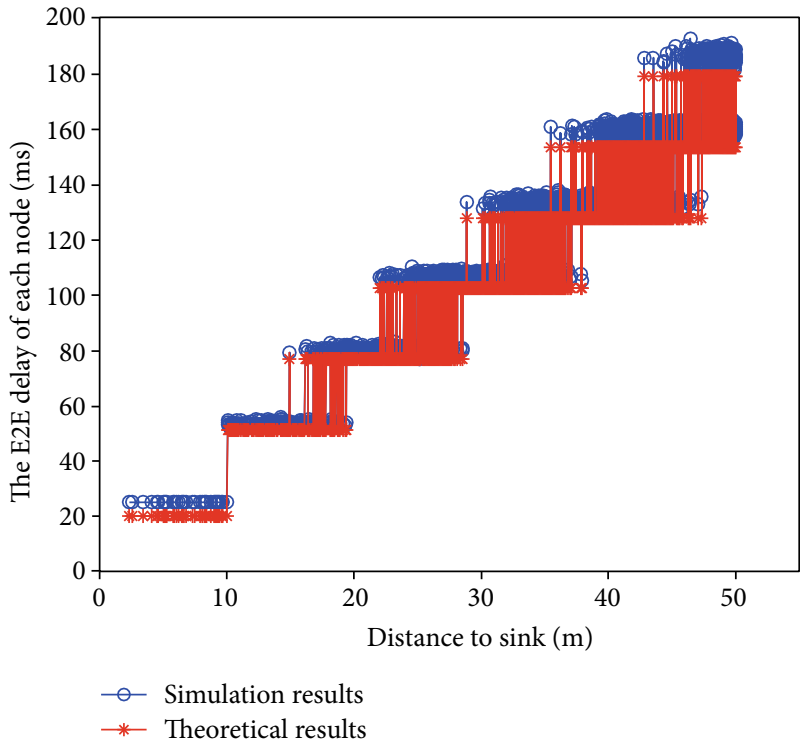

(b)

Figure 8: Comparison of the simulation results and the theoretical analysis in a 3D WSN. (a) The number of sending packet of each node. (b) The E2E delay of each node.

evaluated and analyzed. The performance of the E2E delay, the energy consumption, and the network reliability are estimated under the different $\delta, \alpha, p$, and $r$.

6.3.1. Different Network Partition $\delta$. As described above, the parameter $\delta R$ is used to divide the network into two partitions. Under different $\delta$, the network performance of reliability, energy cost, and E2E delay are presented. The results are demonstrated in Table 2. From this table, we can see that the proposed energy-aware transmission scheme can meet the required transmission reliability $\zeta=0.8$ whether $\delta=0.4$ or $\delta=0.2$. There is more energy consumption for $\delta=0.2$ shown in Table 2. And there is less energy cost for $\delta=0.4$. This is because that there are less nodes adopting the multiple-packet copy transmission and more nodes applying the timeout retransmission scheme when $\delta$ has bigger value. The energy cost of timeout retransmission scheme is less than that of multiple-packet copy transmission scheme under the same network conditions. Therefore, there are less energy cost when $\delta=0.4$ compared with when $\delta=0.2$. For this reason, the E2E delay and the maximal network E2E delay when $\delta=0.4$ is bigger than when $\delta=0.2$ as shown in the table.

6.3.2. Different Number of Returned ACKs $\alpha$. Under different $\alpha$, the network performance of reliability, energy cost, and E2E delay is evaluated. In Table 3, the network reliability under different $\alpha$ is shown. From the table, the required transmission reliability is satisfied. And with the increasing number of ACK, the transmission reliability is improved, and the maximal energy cost of the nodes in one round of data gathering is reduced. The E2E delay is also reduced. This is because that the ACK loss will lead to packet retransmission. Thus, reducing the ACK loss will reduce the unneces-
TABLE 2: Simulation results under different $\delta$ when $p=0.7, \alpha=1$, $r=10$, and $\zeta=0.8$.

\begin{tabular}{lccc}
\hline$\delta$ & Reliability & $\begin{array}{c}\text { The maximal energy } \\
\text { cost }(\mathrm{J})\end{array}$ & $\begin{array}{c}\text { The maximal E2E } \\
\text { delay(ms) }\end{array}$ \\
\hline$\delta=0.2$ & $85.95 \%$ & 0.0043 & 307.5556 \\
$\delta=0.4$ & $85.15 \%$ & 0.0037 & 486.1250 \\
\hline
\end{tabular}

sary packet retransmission. Therefore, a reasonable number of returned ACK will decrease the unnecessary packet retransmission caused by ACK loss.

6.3.3. Different Hop Transmission Reliability $p$. The network performance results of reliability, energy cost, and E2E delay are presented in Table 4 under different $p$. In Table 4 , the transmission reliability is ensured under different one-hop transmission reliability $p$ whether $\delta=0.2$ or $\delta=0.4$. However, the energy cost is bigger with the worse link. The E2E delay is also bigger compared with high reliable transmission link as shown in the table. It is obvious that to meet the transmission reliability constraint, the worse link needs a greater number of packet retransmissions, which cause more energy cost and larger E2E delay.

6.3.4. Different Transmission Radius $r$. Under different $r$, the network performance of reliability, energy cost, and E2E delay are evaluated. Table 5 shows the results. From the table, the transmission reliability is ensured under different $r$. Besides, the results show the overall changing trend of the energy cost of the nodes in one round of data gathering. The overall trend is increasing with smaller distance to the sink. This is because that the nodes near the sink need to 
TABLE 3: Simulation results under different $\alpha$ when $p=0.7, \delta=0.4$, $r=10$, and $\zeta=0.8$.

\begin{tabular}{llcc}
\hline$\alpha$ & Reliability & $\begin{array}{c}\text { The maximal energy } \\
\text { cost }(\mathrm{J})\end{array}$ & $\begin{array}{c}\text { The maximal E2E } \\
\text { delay }(\mathrm{ms})\end{array}$ \\
\hline$\alpha=1$ & $85.15 \%$ & 0.0037 & 486.1250 \\
$\alpha=2$ & $89.64 \%$ & 0.0031 & 419.4186 \\
$\alpha=3$ & $91.76 \%$ & 0.0031 & 379.7674 \\
\hline
\end{tabular}

TABLE 4: Simulation results under different $p$ when $\alpha=1, r=10$, and $\zeta=0.8$.

\begin{tabular}{lcccc}
\hline$p$ & $\delta$ & Reliability & $\begin{array}{c}\text { The maximal } \\
\text { energy cost }(\mathrm{J})\end{array}$ & $\begin{array}{c}\text { The maximal E2E } \\
\text { delay }(\mathrm{ms})\end{array}$ \\
\hline \multirow{2}{*}{$p=0.7$} & $\delta=0.2$ & $85.95 \%$ & 0.0043 & 307.5556 \\
& $\delta=0.4$ & $85.15 \%$ & 0.0037 & 486.1250 \\
\multirow{2}{*}{$p=0.6$} & $\delta=0.2$ & $85.06 \%$ & 0.0053 & 380.2439 \\
& $\delta=0.4$ & $84.37 \%$ & 0.0047 & 670.7317 \\
\hline
\end{tabular}

TABLE 5: Simulation results under different $r$ when $\alpha=1, \delta=0.2, p$ $=0.7$, and $\zeta=0.8$.

\begin{tabular}{lccc}
\hline$r$ & Reliability & $\begin{array}{c}\text { The maximal energy } \\
\text { cost }(\mathrm{J})\end{array}$ & $\begin{array}{c}\text { The maximal E2E } \\
\text { delay }(\mathrm{ms})\end{array}$ \\
\hline$r=10$ & $85.95 \%$ & 0.0043 & 307.5556 \\
$r=12$ & $87.55 \%$ & 0.0054 & 262.5000 \\
$r=15$ & $88.07 \%$ & 0.0037 & 226.9048 \\
\hline
\end{tabular}

forward more packets. Besides, as can be seen from the table, the E2E delay is reduced with the bigger transmission radius. This is because that the bigger radius could reduce the transmission hops to the sink. Also, the number of sending packet copies or the maximal retransmission number is reduced. Therefore, the E2E delay is decreased.

6.3.5. Different Network Reliability $\zeta$. Under different requirements of network reliability $\zeta$, the network performance of achieved reliability, energy cost, and E2E delay are presented in the part. Table 6 shows the network actual reliability achieved under different $\zeta$. From the table, the results show the proposed transmission scheme could meet the different transmission reliability requirements. As can be seen from the table, the energy cost is bigger when the bigger required reliability under different transmission reliability constraints. It is obvious that higher transmission reliability needs the greater number of packet retransmissions under the same wireless link lost. Similarly, the E2E delay also gets bigger as can be seen from the table.

6.4. Performance Comparison. In the section, the performance of the proposed scheme is estimated by comparing with the existing schemes of multiple-packet copies without ACK returned transmission (MP) and timeout retransmis-
TABle 6: Simulation results under different $\zeta$ when $\alpha=1, \delta=0.2, p$ $=0.7$, and $r=10$.

\begin{tabular}{lccc}
\hline$\zeta$ & Reliability & $\begin{array}{c}\text { The maximal energy } \\
\text { cost }(\mathrm{J})\end{array}$ & $\begin{array}{c}\text { The maximal E2E } \\
\text { delay }(\mathrm{ms})\end{array}$ \\
\hline$\zeta=80 \%$ & $85.95 \%$ & 0.0043 & 307.5556 \\
$\zeta=70 \%$ & $81.49 \%$ & 0.0040 & 299.0244 \\
$\zeta=60 \%$ & $64.25 \%$ & 0.0028 & 290.4176 \\
\hline
\end{tabular}

sion scheme represented typically by sending one data packet with one ACK returned (ODOA).

In Figure 9(a), the results demonstrate that the proposed energy-aware transmission scheme can meet the required transmission reliability $\zeta=0.8$. As can be seen from Figures $9(\mathrm{~b})$ and 9(c), there is more energy consumption for MP scheme. And there is less energy cost for ODOA scheme. The energy cost of the proposed transmission scheme is less than MP and bigger than ODOA from the overall perspective. From Figures 9(d) and 9(e), we can see that the delay is bigger with the greater distance of the nodes to the sink overall. The maximum delay of the proposed transmission scheme is bigger than the MP and smaller than the ODOA. Compared with MP, the maximum energy cost is reduced by $29.13 \%$ and $17.23 \%$, respectively, for the proposed scheme when $\delta=0.2$ and $\delta=0.4$. Comparing the proposed scheme with the ODOA, the maximum delay is, respectively, reduced by $43.60 \%$ and $64.32 \%$ when $\delta=0.2$ and $\delta=0.4$. Therefore, the proposed scheme can realize the trade-off optimization between energy efficiency and E2E delay.

\section{Conclusions}

In the paper, the data loads of each node in 3D WSN are analyzed in mathematical model. The energy cost is unbalanced. For the nodes far from the sink, the energy cost is less. However, there are more energy costs for the nodes near the sink. Based on the analysis, an energy-aware transmission scheme is proposed for $3 \mathrm{D}$ WSN to achieve trade-off optimization between E2E delay and energy efficiency under the guarantee of transmission reliability. For nodes far from the sink, they send or forward multiple-packet copies consecutively without waiting for ACK. This could reduce the one-hop transmission delay and improve the energy efficiency under the guarantee of the network transmission reliability. To save energy cost, the nodes near the sink send one packet and then wait for the returned ACK. If the source or relay nodes receive the ACK, the packet is successfully transmitted to the next hop. Otherwise, the nodes will retransmit the packet when timeout.

However, the correlation efficient between the simulation and theoretical results in the mathematical analysis is not high enough. And the contention model is not considered in the proposed scheme. To improve the theoretical analysis accuracy and combine the contention model to reduce the 


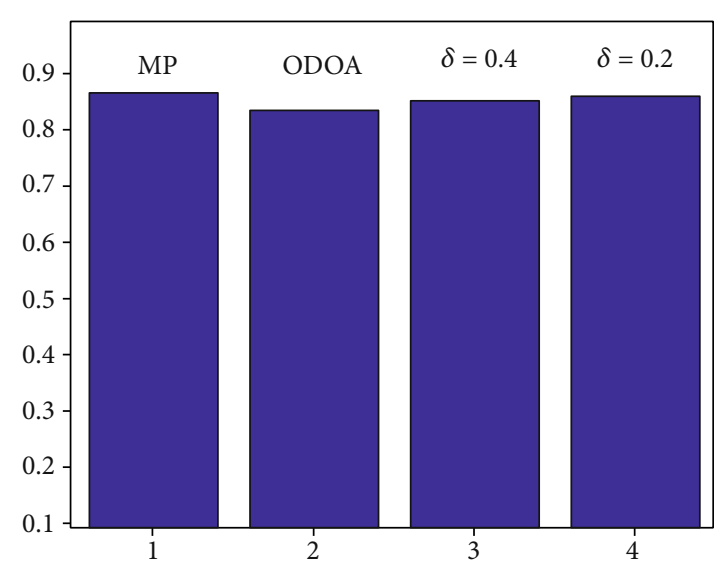

(a)

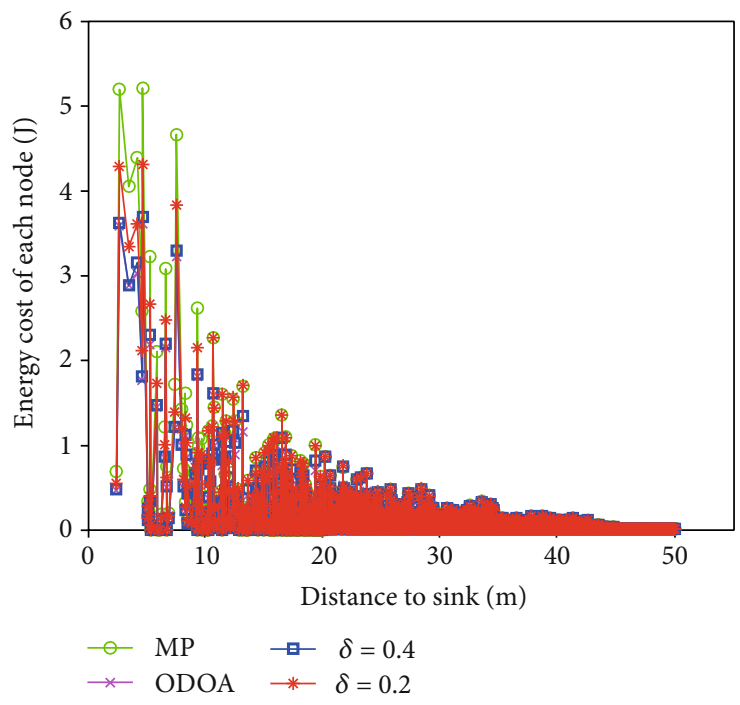

(c)

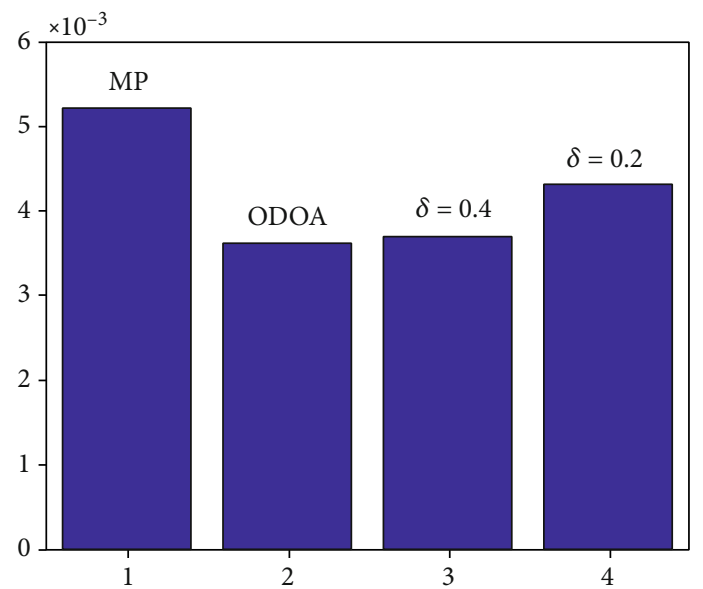

(b)

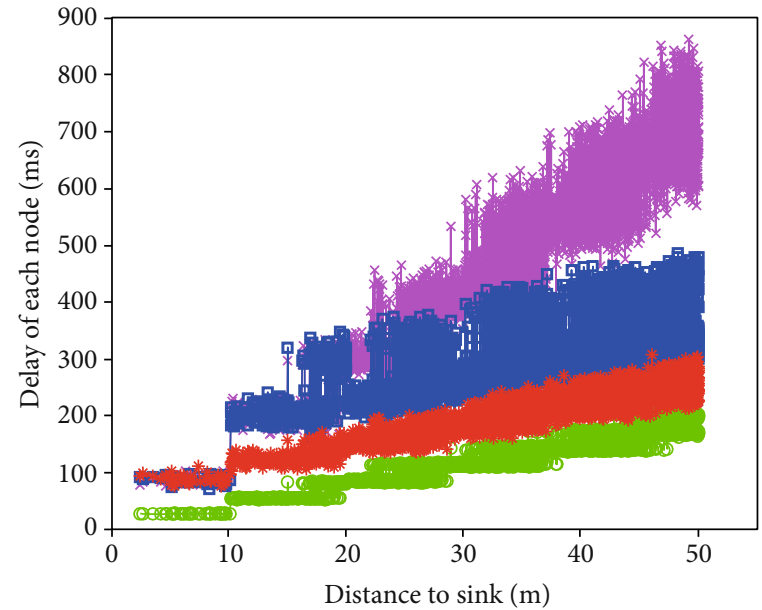

(d)

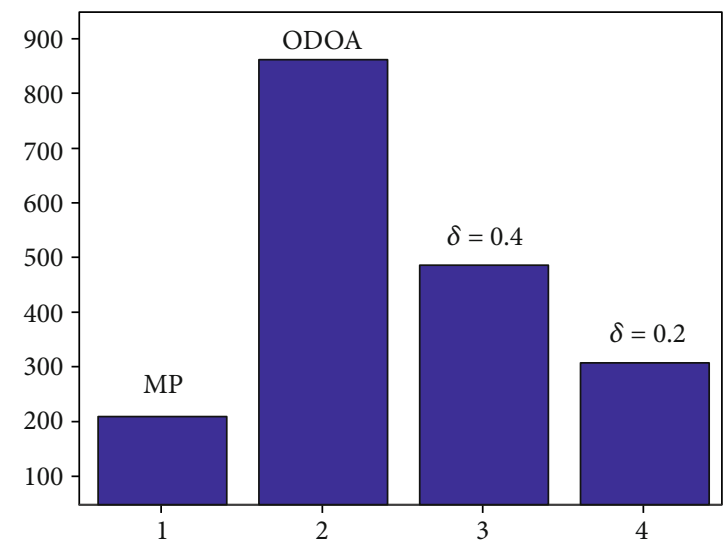

(e)

FIGURE 9: Comparison between schemes under $\alpha=1, p=0.7$, and $r=10$. (a) The network reliability. (b) The energy cost of each node. (c) The maximal energy consumption. (d) The E2E delay of each node. (e) The maximal E2E delay. 
energy cost and E2E delay under the transmission reliability constraint is our further work.

\section{Data Availability}

The generated or analysed data used to support the findings of this study are included within the article.

\section{Conflicts of Interest}

The authors declared no potential conflicts of interest with respect to the research, authorship, and/or publication of this article.

\section{Acknowledgments}

This work was supported in part by the National Natural Science Foundation under grant 61902432 and the Innovation Driven Plan of Central South University under grant 2019CX008.

\section{References}

[1] J. M. Perkel, "The Internet of things comes to the lab," Nature, vol. 542, no. 7639, pp. 125-126, 2017.

[2] Q. A. Ju and Y. Zhang, "Clustered data collection for Internet of batteryless things," IEEE Internet of Things Journal, vol. 4, no. 6, pp. 2275-2285, 2017.

[3] V. Ozdemir and N. Hekim, "Birth of industry 5.0: making sense of big data with artificial intelligence, "the Internet of things" and next-generation technology policy," OMICS-A Journal of Integrative Biology, vol. 22, no. 1, pp. 65-76, 2018.

[4] S. E. Yoo, A. M. K. Cheng, and P. K. Chong, "Technological advances in wireless sensor networks enabling diverse Internet of things applications," International Journal of Distributed Sensor Networks, vol. 14, no. 3, 2018.

[5] M. Gidlund, S. Han, and E. Sisinni, "Guest Editorial From industrial wireless sensor networks to industrial Internet of things," IEEE Transactions on Industrial Informatics, vol. 14, no. 5, pp. 2194-2198, 2018.

[6] Z. Zhao, J. Wang, C. Fu, Z. Liu, D. Liu, and B. Li, "Design of a smart sensor network system for real-time air quality monitoring on green roof," Journal of Sensors, vol. 2018, Article ID 1987931, 13 pages, 2018.

[7] X. B. Min and W. H. Kuang, "Study on the ecological farming control system based on the Internet of things," Wireless Personal Communications, vol. 102, no. 4, pp. 2955-2967, 2018.

[8] J. L. LeeL, Y. Y. Tyan, and M. H. Wen, "Applying ZigBee wireless sensor and control network for bridge safety monitoring," Advances in Mechanical Engineering, vol. 10, no. 7, 2018.

[9] C. Zhou, H. B. Luo, and W. L. Fang, "Cyber-physical-systembased safety monitoring for blind hoisting with the Internet of things: a case study," Automation in Construction, vol. 97, pp. 138-150, 2019.

[10] C. Habib, A. Makhoul, R. Darazi, and R. Couturier, "Health risk assessment and decision-making for patient monitoring and decision-support using wireless body sensor networks," Information Fusion, vol. 47, pp. 10-22, 2019.

[11] A. H. Mohsin, A. A. Zaidan, B. B. Zaidan et al., "Real-time remote health monitoring systems using body sensor information and finger vein biometric verification: a multi-layer sys- tematic review," Journal of Medical Systems, vol. 42, no. 12, 2018.

[12] Y. Y. Shih, P. C. Hsiu, and A. C. Pang, "A data parasitizing scheme for effective health monitoring in wireless body area networks," IEEE Transactions on Mobile Computing, vol. 18, no. 1, pp. 13-27, 2019.

[13] F. Ding, A. Song, D. Zhang, E. Tong, Z. Pan, and X. You, "Interference-aware wireless networks for home monitoring and performance evaluation," IEEE Transactions on Automation Science and Engineering, vol. 15, no. 3, pp. 1286-1297, 2018.

[14] O. Blanco-Novoa, T. Fernández-Caramés, P. Fraga-Lamas, and L. Castedo, "A cost-effective IoT system for monitoring indoor radon gas concentration," Sensors, vol. 18, no. 7, p. 2198, 2018.

[15] B. Jiang, G. Huang, T. Wang, J. Gui, and X. Zhu, “Trust based energy efficient data collection with unmanned aerial vehicle in edge network," Transactions on Emerging Telecommunications Technologies, 2020.

[16] J. Li, D. Su, and Y. Wang, "Energy-efficient and traffic-adaptive z-medium access control protocol in wireless sensor networks," IET Wireless Sensor Systems, vol. 8, no. 5, pp. 208$214,2018$.

[17] L. Muduli, D. P. Mishra, and P. K. Jana, "Application of wireless sensor network for environmental monitoring in underground coal mines: a systematic review," Journal of Network and Computer Applications, vol. 106, pp. 48-67, 2018.

[18] F. Li, Z. Chen, and Y. Wang, "Localized geometric topologies with bounded node degree for three-dimensional wireless sensor networks," Eurasip Journal on Wireless Communications and Networking, vol. 2012, no. 1, 2012.

[19] L. Yi and M. Chen, "An enhanced hybrid 3D localization algorithm based on APIT and DV-Hop," International Journal of Online Engineering, vol. 13, no. 9, pp. 69-86, 2017.

[20] K. Munasinghe, M. Aseeri, S. Almorqi, M. F. Hossain, M. Binte Wali, and A. Jamalipour, "EM-based High Speed wireless sensor networks for underwater surveillance and target tracking," Journal of Sensors, vol. 2017, 14 pages, 2017.

[21] W. Y. Cai and M. Y. Zhang, "3D Dubins curves based path programming for mobile sink in underwater sensor networks," Electronics Letters, vol. 53, no. 1, pp. 48-50, 2017.

[22] G. Han, S. Shen, H. Song, T. Yang, and W. Zhang, "A stratification-based data collection scheme in underwater acoustic sensor networks," IEEE Transactions on Vehicular Technology, vol. 67, no. 11, pp. 10671-10682, 2018.

[23] A. Liu, Z. Liu, M. Nurudeen, X. Jin, and Z. Chen, “An elaborate chronological and spatial analysis of energy hole for wireless sensor networks," Computer Standards \& Interfaces, vol. 35, no. 1, pp. 132-149, 2013.

[24] J. Ren, Y. Zhang, K. Zhang, A. Liu, J. Chen, and X. S. Shen, "Lifetime and energy hole evolution analysis in datagathering wireless sensor networks," IEEE Transactions on Industrial Informatics, vol. 12, no. 2, pp. 788-800, 2016.

[25] A. Liu, X. Jin, G. Cui, and Z. Chen, "Deployment guidelines for achieving maximum lifetime and avoiding energy holes in sensor network," Information Sciences, vol. 230, pp. 197-226, 2013.

[26] D. L. Donoho, "Compressed sensing," IEEE Transactions on Information Theory, vol. 52, no. 4, pp. 1289-1306, 2006.

[27] E. J. Candes and M. B. Wakin, "An introduction to compressive sampling," IEEE Signal Processing Magazine, vol. 25, no. 2, pp. 21-30, 2008. 
[28] J. Tan, W. Liu, T. Wang, M. Zhao, A. Liu, and S. Zhang, "A high-accurate content popularity prediction computational modeling for mobile edge computing using matrix completion technology," Transactions on Emerging Telecommunications Technologies, 2020.

[29] M. Huang, K. Zhang, Z. Zeng, T. Wang, and Y. Liu, “An AUVassisted data gathering scheme based on clustering and matrix completion for smart ocean," IEEE Internet of Things Journal, 2020.

[30] P. G. V. Naranjo, M. Shojafar, H. Mostafaei, Z. Pooranian, and E. Baccarelli, "P-SEP: a prolong stable election routing algorithm for energy-limited heterogeneous fog-supported wireless sensor networks," Journal of Supercomputing, vol. 73, no. 2, pp. 733-755, 2017.

[31] D. T. Hai, L. H. Son, and T. Le Vinh, "Novel fuzzy clustering scheme for 3D wireless sensor networks," Applied Soft Computing, vol. 54, pp. 141-149, 2017.

[32] D. Y. Ye and M. J. Zhang, "A self-adaptive sleep/wake-up scheduling approach for wireless sensor networks," IEEE Transactions on Cybernetics, vol. 48, no. 3, pp. 979-992, 2018.

[33] J. F. Myoupo, B. P. Nana, and V. K. Tchendji, "Fault-tolerant and energy-efficient routing protocols for a virtual threedimensional wireless sensor network," Computers and Electrical Engineering, vol. 72, pp. 949-964, 2018.

[34] M. S. Gharajeh and S. Khanmohammadi, "DFRTP: dynamic 3D fuzzy routing based on traffic probability in wireless sensor networks," IET Wireless Sensor Systems, vol. 6, no. 6, pp. 211219, 2016.

[35] P. Gupta and P. R. Kumar, "The capacity of wireless networks," IEEE Transaction on Information Theory, vol. 46, no. 2, pp. 388-404, 2000.

[36] Y. X. Liu, A. F. Liu, and Z. G. Chen, "Analysis and improvement of send-and-wait automatic repeat-request protocols for wireless sensor networks," Wireless Personal Communications, vol. 81, no. 3, pp. 923-959, 2015. 